Kragujevac Journal of Mathematics

Volume 45(6) (2021), Pages 881-896.

\title{
ON THE APPLICATIONS OF BOCHNER-KODAIRA-MORREY-KOHN IDENTITY
}

\author{
SAYED SABER ${ }^{1,2}$
}

\begin{abstract}
This paper is devoted to studying some applications of the BochnerKodaira-Morrey-Kohn identity. For this study, we define a condition which is called $\left(H_{q}\right)$ condition which is related to the Levi form on the complex manifold. Under the $\left(H_{q}\right)$ condition and combining with the basic Bochner-Kodaira-Morrey-Kohn identity, we study the $L^{2} \bar{\partial}$ Cauchy problems on domains in $\mathbb{C}^{n}$, Kähler manifold and in projective space. Also, we study this problem on a piecewise smooth strongly pseudoconvex domain in a complex manifold. Furthermore, the weighted $L^{2} \bar{\partial}$ Cauchy problem is studied under the same condition in a Kähler manifold with semi-positive holomorphic bisectional curvature. On the other hand, we study the global regularity and the $L^{2}$ theory for the $\bar{\partial}$-operator with mixed boundary conditions on an annulus domain in a Stein manifold between an inner domain which satisfy $\left(H_{n-q-1}\right)$ and an outer domain which satisfy $\left(H_{q}\right)$.
\end{abstract}

\section{INTRODUCTION}

$\bar{\partial}$-equation has been a powerful method in complex geometry and complex algebraic geometry. The pioneer works of $\bar{\partial}$-equation are due to Kohn and Hörmander on the existence and boundary regularity of $\bar{\partial}$-equation on pseudoconvex domain. The $L^{2}$ estimate is a powerful method when solving $\bar{\partial}$-equation. In order to establish the $L^{2}$ estimate for $\bar{\partial}$ operator, a crucial step is to obtain a basic identity which is due to Bochner, Kodaira, Morrey, Kohn and Hörmander. Then after imposing some conditions such as the curvature of the complex vector bundle and the Levi form of the complex manifold, one can get a priori estimate which we call $L^{2}$ estimate for the $\bar{\partial}$ operator.

Key words and phrases. $\bar{\partial}, \bar{\partial}$-Neumann operator, weakly $q$-convex domains.

2010 Mathematics Subject Classification. Primary: 32F10, 32W05. Secondary: 32W10, 35J20, $35 \mathrm{~J} 60$.

DOI 10.46793/KgJMat2106.881S

Received: November 06, 2018.

Accepted: June 10, 2019. 
In 1940, Bochner introduced his technique for getting topological information from the behavior of harmonic forms. In the late of 1950 and early of 1960, the approaches of Bochner-Kodaira and Oka is very deep approach based in partial differential equations. In 1966, Spencer defined the $\bar{\partial}$-Neumann problem and Kohn who finally formulated and solved the $\bar{\partial}$-Neumann problem on strictly pseudoconvex domains. Shortly after Kohn's work, Hörmander and Andreotti-Vesentini, independently and almost simultaneously, obtained weighted $L^{2}$ estimates for the inhomogeneous Cauchy-Riemann equations.

The paper consists of two parts. In the first part, we study the Cauchy-Riemann equations with compact support ( $L^{2}$ Cauchy problem) on a domain in $\mathbb{C}^{n}$ which satisfy property $\left(H_{q}\right)$. Moreover, we extend this result to the same domain in Kähler manifold for vector-valued forms of type $(r, q), q \geqslant 1$. Also, we study this problem on a piecewise smooth strongly pseudoconvex domain in a complex manifold. Furthermore, the weighted $L^{2} \bar{\partial}$ Cauchy problem is studied on the same domains in an $n$-dimensional Kähler manifold with semi-positive holomorphic bisectional curvature. In the papers of Kohn-Rossi [13], Cao-Shaw-Wang [2], Abdelkader-Saber [1] and Saber [18]-[21] such equations are studying for various domains.

In the second part of this paper, we study the $\bar{\partial}$ equation on domains with mixed boundary conditions which was studied by Li and Shaw [16]. We generalize Li-Shaw's result to annulus in Stein manifolds under the conditions $\left(H_{q}\right)$ defined here. Namely, we study the global boundary regularity and the $L^{2}$ theory for $\bar{\partial}_{m i x}$-operator on an annulus domain in a Stein manifold between an internal domain which satisfies condition $\left(H_{n-q-1}\right)$ and an external domain which satisfies condition $\left(H_{q}\right)$. Making use of the method developed by Catlin, we study the $L^{2}$-estimate for the $\bar{\partial}_{m i x}$-equation with mixed boundary conditions. This equation with various boundary conditions are the basic tools to work on analytic problems in several complex variables and complex geometry or many geometric. In the papers of Catlin [3], Cho [6] and Catlin-Cho [4], such equations played a crucial role for studying various extension problems for CR structures. In a paper of Catlin [3], Catlin proved that there is no cohomology obstruction for solving the $\bar{\partial}_{m i x}$-equations. Other related studies for the $\bar{\partial}$-Dirichlet problem can be found in the work of Li-Shaw [16] and Huang-Li [11].

\section{2. $L^{2} \bar{\partial}$ Cauchy Problem in $\mathbb{C}^{n}$}

Let $0 \leqslant r, q \leqslant n$, we can write an arbitrary $(r, q)$-form $f$ as $f=\sum_{I, J}^{\prime} f_{I, J} d z^{I} \wedge d \bar{z}^{J}$, where $I=\left(i_{1}, \ldots, i_{r}\right)$ and $J=\left(j_{1}, \ldots, j_{q}\right)$ are multiindices and $d z^{I}=d z_{i_{1}} \wedge \cdots \wedge d z_{i_{r}}$, $d \bar{z}^{J}=d \bar{z}_{j_{1}} \wedge \cdots \wedge d \bar{z}_{j_{q}}$. The notation $\sum^{\prime}$ means the summation over strictly increasing multiindices. Let $\Omega$ be a relatively compact domain in $\mathbb{C}^{n}$ and let $C_{r, q}^{\infty}\left(\mathbb{C}^{n}\right)$ be the complex vector space of complex-valued differential $(r, q)$-forms of class $C^{\infty}$ on $\mathbb{C}^{n}$. Let $C_{r, q}^{\infty}(\bar{\Omega})=\left\{\left.f\right|_{\bar{\Omega}}: f \in C_{r, q}^{\infty}\left(\mathbb{C}^{n}\right)\right\}$ be the subspace of $C_{r, q}^{\infty}(\Omega)$ whose elements can be extended smoothly up to the boundary $b \Omega$. Let $\phi: \mathbb{C}^{n} \rightarrow \mathbb{R}^{+}$be a plurisubharmonic 
$C^{2}$-weight function and define the space

$$
L^{2}(\Omega, \phi)=\left\{f: \Omega \longrightarrow \mathbb{C}: \int_{\Omega}|f|^{2} e^{-\phi} d V<\infty\right\}
$$

where $d V$ denotes the Lebesgue measure. Denote the inner product and the norm in $L^{2}(\Omega, \phi)$ by

$$
\langle f, g\rangle_{\phi}=\int_{\Omega} f \bar{g} e^{-\phi} d V \text { and }\|f\|_{\phi}=\int_{\Omega}|f|^{2} e^{-\phi} d V .
$$

Recall that $L_{r, q}^{2}(\Omega, \phi)$ the space of $(r, q)$-forms with coefficients in $L^{2}(\Omega, \phi)$. If $f, g \in$ $L_{r, q}^{2}(\Omega, \phi)$, the $L^{2}$-inner product and norms are defined by

$$
\langle f, g\rangle_{\phi}=\int_{\Omega} f \wedge \star \bar{g} e^{-\phi} \text { and }\|f\|_{\phi}^{2}=\langle f, f\rangle_{\phi},
$$

where $\star$ is the Hodge star operator (for detailed discussions of the Hodge star operator in the $L^{2}$-space see [5]).

Let $\bar{\partial}: L_{r, q-1}^{2}(\Omega, \phi) \rightarrow L_{r, q}^{2}(\Omega, \phi)$ be the closed operator which is the maximal extension of the differential operator and $\bar{\partial}^{*}$ be its $L^{2}$-adjoint. Here the $\bar{\partial}$ and $\bar{\partial}^{*}$ operators are defined as

$$
\begin{aligned}
\bar{\partial} f & =\sum_{I, J}^{\prime} \sum_{\beta=1}^{n} \frac{\partial f_{I, J}}{\partial \bar{z}^{\beta}} d \bar{z}^{\beta} \wedge d z^{I} \wedge d \bar{z}^{J} \\
\bar{\partial}^{*} f & =(-1)^{r-1} \sum_{I, K}^{\prime} \sum_{\alpha=1}^{n} \frac{\partial f_{I, \alpha K}}{\partial z^{\alpha}} d z^{I} \wedge d \bar{z}^{K} .
\end{aligned}
$$

If $w \in \operatorname{dom} \bar{\partial}$ and $u \in \operatorname{dom} \bar{\partial}^{*}$, then

$$
\langle\bar{\partial} w, u\rangle_{\phi}=\left\langle\bar{\partial} w, e^{-\phi} u\right\rangle=\left\langle w, \bar{\partial}^{*}\left(e^{-\phi} u\right)\right\rangle=\left\langle w, e^{\phi} \bar{\partial}^{*}\left(e^{-\phi} u\right)\right\rangle_{\phi}
$$

Thus, $\bar{\partial}_{\phi}^{*}=e^{\phi} \bar{\partial}^{*}\left(e^{-\phi}\right)$. The complex Laplacian on $(r, q)$-forms is defined as $\square_{r, q}=$ $\bar{\partial} \bar{\partial}^{*}+\bar{\partial}^{*} \bar{\partial}$, for $q \geqslant 1$ and the $\bar{\partial}$-Neumann operator $N_{r, q}$ is - if it exists-the bounded inverse of $\square_{r, q}$. Following $\left(\left[12, \mathrm{I}\right.\right.$, page 127]), we set $\mathcal{B}_{r, q}(\bar{\Omega})=C_{r, q}^{\infty}(\bar{\Omega}) \cap \operatorname{dom} \bar{\partial}^{*}$. Let $\mathcal{B}_{r, q}(U)$ denote the subset of $\mathcal{B}_{r, q}(\bar{\Omega})$ consisting of those forms whose support lies in $U \cap \bar{\Omega}$. We define the following norms on $\mathcal{B}_{r, q}(U)$ :

$$
\begin{aligned}
& E(u)=\sum_{I, J}^{\prime} \sum_{k=1}^{n} \int_{\Omega}\left|\frac{\partial u_{I, J}}{\partial \bar{z}^{k}}\right|^{2} d V+\|u\|^{2}, \\
& D(u)=\|\bar{\partial} u\|^{2}+\left\|\bar{\partial}^{*} u\right\|^{2}+\|u\|^{2} .
\end{aligned}
$$

Definition 2.1. A bounded domain $\Omega$ with $C^{2}$-smooth boundary is said to be satisfy condition $\left(H_{q}\right)$ if there exists a defining function $\delta$ for $\Omega$ such that the sum $s_{q}$ of any $q$ eigenvalues of the matrix $\left(\frac{\partial^{2} \delta}{\partial z^{\alpha} \partial \bar{z}^{\beta}}\right)$ of the Levi form $\partial \bar{\partial} \delta(z)$ is semi-positive on $\bar{\Omega}$.

Proposition 2.1 (Bochner-Hörmander-Kohn-Morrey formula). Let $\Omega$ be a bounded domain in $\mathbb{C}^{n}$ with defining function $\delta$ and let $\psi, \phi$ be two real functions that are twice 
continuously differentiable on $\bar{\Omega}$, with $\psi \geqslant 0$. Then, for $u \in \mathcal{B}_{r, q}(\bar{\Omega})$ with $1 \leqslant q \leqslant n-1$, we have

$$
\begin{aligned}
& \|\sqrt{\psi} \bar{\partial} u\|_{\phi}^{2}+\left\|\sqrt{\psi} \bar{\partial}_{\phi}^{*} u\right\|_{\phi}^{2} \\
= & \sum_{I, K}^{\prime} \sum_{\alpha, \beta=1}^{n} \int_{b \Omega} \psi \frac{\partial^{2} \delta}{\partial z^{\alpha} \partial \bar{z}^{\beta}} u_{I, \alpha K} \bar{u}_{I, \beta K} e^{-\phi} d S \\
& +\sum_{I, J}^{\prime} \sum_{k=1}^{n} \int_{\Omega} \psi\left|\frac{\partial u_{I, J}}{\partial \bar{z}^{k}}\right|^{2} e^{-\phi} d V+2 \operatorname{Re}\left(\sum_{I, K}^{\prime} \sum_{\alpha=1}^{n} \frac{\partial \psi}{\partial z^{\alpha}} u_{I, \alpha K} d z^{I} \wedge d \bar{z}^{K}, \bar{\partial}_{\phi}^{*} u\right)_{\phi} \\
& +\sum_{I, K}^{\prime} \sum_{\alpha, \beta=1}^{n} \int_{\Omega}\left(\psi \frac{\partial^{2} \phi}{\partial z^{\alpha} \partial \bar{z}^{\beta}}-\frac{\partial^{2} \psi}{\partial z^{\alpha} \partial \bar{z}^{\beta}}\right) u_{I, \alpha K} \bar{u}_{I, \beta K} e^{-\phi} d V .
\end{aligned}
$$

Remark 2.1. When $\psi \equiv 1$ and $\phi \equiv 0$, one obtains the classical Kohn-Morrey formula ([12] and [9]).

Proposition 2.2. Let $\Omega \Subset \mathbb{C}^{n}$ be a bounded domain with $C^{2}$-smooth boundary satisfying condition $\left(H_{q}\right)$. Then, for $u \in \mathcal{B}_{r, q}(\bar{\Omega})$, one obtains

$$
E(u) \leqslant C D(u) \text {. }
$$

Moreover, there exists a uniquely determined bounded linear operator $N_{r, q}: L_{r, q}^{2}(\Omega) \rightarrow$ $L_{r, q}^{2}(\Omega)$, such that $\square_{r, q} \circ N_{r, q} u=u$ for any $u \in L_{r, q}^{2}(\Omega)$.

Proof. Let $\mu_{1} \leqslant \mu_{2} \leqslant \cdots \leqslant \mu_{n}$ denote the eigenvalues of the matrix $\left(\frac{\partial^{2} \delta}{\partial z^{\alpha} \partial \bar{z}^{\beta}}\right)$ and suppose that $\left(\frac{\partial^{2} \delta}{\partial z^{\alpha} \partial \bar{z}^{\beta}}\right)$ is diagonalized. Then, in a suitable basis,

$$
\begin{aligned}
\sum_{I, K}^{\prime} \sum_{\alpha, \beta=1}^{n-1} \frac{\partial^{2} \delta}{\partial z^{\alpha} \partial \bar{z}^{\beta}} u_{I, \alpha K} \overline{u_{I, \beta K}} & =\sum_{I, K}^{\prime} \sum_{\alpha=1}^{n} \mu_{\alpha}\left|u_{I, \alpha K}\right|^{2} \\
& =\sum_{\substack{|I|=r \\
J=\left(j_{1}, j_{2}, \ldots, j_{q}\right)}}^{\prime}\left(\mu_{1}+\mu_{2}+\cdots+\mu_{q}\right)\left|u_{I, J}\right|^{2} \geqslant s_{q}|u|^{2} .
\end{aligned}
$$

The second equality follows as. For $J=\left(j_{1}, j_{2}, \ldots, j_{q}\right)$ fixed, $\left|u_{I, J}\right|^{2}$ occurs precisely $q$ times in the second sum, once as $\left|u_{I, j_{1} K}\right|^{2}$, once as $\left|u_{I, j_{2} K}\right|^{2}$, etc. At each occurrence, it is multiplied by $\mu_{j l}$. By fixing $\left(j_{1}, j_{2}, \ldots, j_{q}\right)$ and set $u=d \bar{z}_{j_{1}} \wedge \cdots \wedge d \bar{z}_{j_{q}}$, we obtain

$$
\sum_{I, K}^{\prime} \sum_{\alpha, \beta=1}^{n-1} \frac{\partial^{2} \delta}{\partial z^{\alpha} \partial \bar{z}^{\beta}} u_{I, \alpha K} \bar{u}_{I, \beta K} \geqslant 0
$$

Thus, the boundary integral in (2.2) is semi-positive. Also, by taking $\phi \equiv 0$ and replace $\psi$ by $1-e^{\lambda}$, where $\lambda$ is an arbitrary twice continuously differentiable nonpositive function, and after applying the Cauchy-Schwarz inequality to the term in (2.2) involving first derivatives of $\psi$, we find

$$
\left\|\sqrt{1-e^{\lambda}} \bar{\partial} u\right\|^{2}+\left\|\sqrt{1-e^{\lambda}} \bar{\partial}^{*} u\right\|^{2} \geqslant \sum_{I, K}^{\prime} \sum_{\alpha, \beta=1}^{n} \int_{\Omega} e^{\lambda} \frac{\partial^{2} \lambda}{\partial z^{\alpha} \partial \bar{z}^{\beta}} u_{I, \alpha K} \bar{u}_{I, \beta K} d V-\left\|e^{\lambda / 2} \bar{\partial}^{*} u\right\|^{2} .
$$


Since $\psi+e^{\lambda}=1$ and $\psi \leqslant 1$, it follows that

$$
\|\bar{\partial} u\|^{2}+\left\|\bar{\partial}^{*} u\right\|^{2} \geqslant \sum_{I, K}^{\prime} \sum_{\alpha, \beta=1}^{n} \int_{\Omega} e^{\lambda} \frac{\partial^{2} \lambda}{\partial z^{\alpha} \partial \bar{z}^{\beta}} u_{I, \alpha K} \bar{u}_{I, \beta K} d V
$$

for every twice continuously differentiable non-positive function $\lambda$. Let $z_{0}$ be a point of $\Omega$, and set $\lambda(z)=-1+\left|z-z_{0}\right|^{2} / d^{2}$, where $d=\sup _{z, z^{\prime} \in \Omega}\left|z-z^{\prime}\right|$ is the diameter of the bounded domain $\Omega$. The preceding inequality then implies the fundamental estimate (2.3) which implies the following estimate

$$
\|u\|^{2} \leqslant \frac{d^{2} e}{q}\left(\|\bar{\partial} u\|^{2}+\left\|\bar{\partial}^{*} u\right\|^{2}\right) .
$$

Then, a bounded linear operator $N_{r, q}: L_{r, q}^{2}(\Omega) \rightarrow L_{r, q}^{2}(\Omega)$ exists, such that $\square_{r, q} \circ$ $N_{r, q} u=u$, for any $u \in L_{r, q}^{2}(\Omega)$.

Theorem 2.1. Let $\Omega \Subset \mathbb{C}^{n}$ be a bounded domain with $C^{2}$-smooth boundary satisfying condition $\left(H_{q}\right)$. For $f \in L_{r, q}^{2}\left(\mathbb{C}^{n}\right), 1 \leqslant q \leqslant n-1$, supp $f \subset \bar{\Omega}$, satisfying $\bar{\partial} f=0$ in the distribution sense in $\mathbb{C}^{n}$, there exists $u \in L_{r, q-1}^{2}\left(\mathbb{C}^{n}\right)$, supp $u \subset \bar{\Omega}$ such that $\bar{\partial} u=f$ in the distribution sense in $\mathbb{C}^{n}$.

Proof. Let $f \in L_{r, q}^{2}\left(\mathbb{C}^{n}\right)$, supp $f \subset \bar{\Omega}$, then $f \in L_{r, q}^{2}(\Omega)$. From Proposition 1.2, $N_{n-r, n-q}$ exists for $n-q \geqslant 1$. Since

$$
u=-\star \overline{\bar{\partial}} N_{n-r, n-q} \star \bar{f} .
$$

Thus, supp $u \subset \bar{\Omega}$ and $u$ vanishes on $b \Omega$. Now, we extend $u$ to $\mathbb{C}^{n}$ by defining $u=0$ in $\mathbb{C}^{n} \backslash \Omega$. As in Saber [18], the extended form $u$ satisfies the equation $\bar{\partial} u=f$ in the distribution sense in $\mathbb{C}^{n}$.

\section{The $L^{2} \bar{\partial}$ Cauchy Problem for Vector-Valued Forms}

Let $X$ be an $n$-dimensional complex manifold with a Hermitian metric $g$. We assume that there is a smooth, real-valued function $\delta$ defined on a neighborhood $U$ of $b \Omega$ in $X$. Assume that $E$ is a holomorphic vector bundle, of rank $p$, over $X$ and $E^{*}$ its dual. An $E$-valued differential $(r, q)$-form $u$ on $X$ is given locally by a column vector ${ }^{t} u=\left(u^{1}, u^{2}, \ldots, u^{p}\right)$, where $u^{a} ; 1 \leqslant a \leqslant p$, are $\mathbb{C}$-valued differential forms of type $(r, q)$ on $X$. For an orthonormal basis $e_{1}, e_{2}, \ldots, e_{p}$ on the fiber $E_{z}=\pi^{-1}(z)$, over $z$, a Hermitian metric $h$ along the fibers of $E$ is expressed as $h=\left(h_{a \bar{b}}\right) ; h_{a \bar{b}}=h\left(e_{a}, e_{b}\right)$. Let $\theta$ be the connection of the Hermitian metric $h(\theta$ is given locally by the $(1,0)$-form $\left.h^{-1} \partial h\right)$. The space $L_{r, q}^{2}(\Omega, E)$ of square integrable differential forms of type $(r, q)$ on $\Omega$ is a Hilbert space under the scalar product

$$
\langle u, v\rangle=\int_{\Omega}{ }^{t}((h) u) \wedge \star \bar{v}=\sum_{a=1}^{p} \int_{\Omega}((h) u)^{a} \wedge \star \overline{(v)^{a}} .
$$

Let $\operatorname{ker}(\bar{\partial}, E)=\{u \in \operatorname{dom}(\bar{\partial}, E): \bar{\partial} u=0\}$ and Range $(\bar{\partial}, E)=\{\bar{\partial} u: u \in$ $\operatorname{dom}(\bar{\partial}, E)\}$ be the kernel and the range of $\bar{\partial}$, respectively. Let $\#_{E}: C_{r, q}^{\infty}(X, E) \rightarrow$ 
$C_{q, r}^{\infty}\left(X, E^{*}\right)$ be the operator defined by $\#_{E} u=\bar{h} \bar{u}$, which commutes with the Hodge star operator. The corresponding operator $\#_{E^{*}}: C_{r, q}^{\infty}\left(X, E^{*}\right) \rightarrow C_{q, r}^{\infty}(X, E)$ is defined by $\#_{E^{*}} u=h^{-1}-u=\#_{E}^{-1} u$. Let $\Pi_{r, q}: L_{r, q}^{2}(\Omega, E) \rightarrow \operatorname{ker}\left(\square_{r, q}, E\right)$ be the orthogonal projection from the space $L_{r, q}^{2}(\Omega, E)$ onto the space $\operatorname{ker}\left(\square_{r, q}, E\right)$. Let $C_{r, q}^{\infty}(\bar{\Omega}, E)=\left\{\left.\varphi\right|_{\bar{\Omega}}: u \in C_{r, q}^{\infty}(X, E)\right\}$ be the subspace of $C_{r, q}^{\infty}(\Omega, E)$ whose elements can be extended smoothly up to $b \Omega$. As in [12, I, page 127], that even for vector bundles, we set $\mathcal{B}_{r, q}(\bar{\Omega}, E)=C_{r, q}^{\infty}(\bar{\Omega}, E) \cap \operatorname{dom}\left(\bar{\partial}^{*}, E\right)$. Let $\mathcal{B}_{r, q}(U, E)$ denote the subset of $\mathcal{B}_{r, q}(\bar{\Omega}, E)$ consisting of those forms whose support lies in $U \cap \bar{\Omega}$. For each $\tau \geqslant 0$, we define the following norms on $\mathcal{B}_{r, q}(U, E)$ :

$$
\begin{aligned}
\|u\|_{\tau}^{2} & =\left\langle u, e^{\tau|\delta|} u\right\rangle, \\
\widetilde{D}_{\tau}^{2}(u) & =\|\bar{\partial} u\|_{\tau}^{2}+\left\|\bar{\partial}_{\tau}^{*} u\right\|_{\tau}^{2}+\|u\|_{\tau}^{2}, \\
\widetilde{E}_{\tau}(u) & =\sum_{a=1}^{p} E_{\tau}\left(u^{a}\right),
\end{aligned}
$$

where $E_{\tau}$ is defined by (2.1) for complex-valued forms.

Theorem 3.1. Let $X$ be an $n$-dimensional complex manifold and let $\Omega \Subset X$ be a bounded domain with $C^{2}$-smooth boundary satisfying condition $\left(H_{q}\right)$. Let $E \rightarrow X$ be a holomorphic vector bundle over $X$. Then, for all $\tau \geqslant 0$, and $u=\left(u^{1}, u^{2}, \ldots, u^{p}\right) \in$ $\mathcal{B}_{r, q}(U, E)$, we obtain

$$
\widetilde{E}_{\tau}^{2}(u)+\tau\|u\|_{\tau}^{2} \leqslant C\left(\widetilde{D}_{\tau}^{2}(u)+\tau^{2} \sum_{\substack { a=1 \\
\begin{subarray}{c}{|I|=r \\
n \in J{ a = 1 \\
\begin{subarray} { c } { | I | = r \\
n \in J } }\end{subarray}}^{\prime}\left\|u_{I J}^{a}\right\|_{\tau}^{2}\right) .
$$

Proof. First, observe that for elements $u=\left(u^{1}, u^{2}, \ldots, u^{p}\right) \in \mathcal{B}_{r, q}(E)$ which have support in $U$, the norms $\|u\|_{\tau}^{2}$ and $\sum_{a=1}^{b}\left\|u^{a}\right\|_{\tau}^{2}$ are equivalent (independently of $\tau$ ). If the metric $h$ is represented by the matrix $\left(h_{a b}\right)$, then

$$
\|u\|^{2}=\int_{\Omega}{ }^{t}((h) u) \wedge \star \bar{u}=\sum_{a, b=1}^{n} \int_{\Omega} h_{a b} u^{a} \wedge \star \overline{(u)^{b}},
$$

where the $h_{a b}$ can be assumed to be $C^{\infty}$ on $\bar{U}$. Now, for $u \in \mathcal{B}_{r, q}(U, E), u=$ $\left(u^{1}, u^{2}, \ldots, u^{p}\right) \in \mathcal{B}(U, E)$ with $u^{a} \in \mathcal{B}_{r, q}(U), a=1,2, \ldots, p$. From (2.3), one obtains

$$
\widetilde{E}_{\tau}^{2}(u)=\sum_{a=1}^{p} E_{\tau}\left(u^{a}\right) \leqslant C \sum_{a=1}^{p}\left(D_{\tau}^{2}\left(u^{a}\right)+\tau^{2} \sum_{\substack{|I|=r \\ n \in J}}^{\prime}\left\|u_{I J}^{a}\right\|_{\tau}^{2}\right) .
$$

Now, since $\theta$ is a $C^{\infty}$ form on $\bar{U}$ and $\vartheta_{\tau} u=\vartheta u-\star \theta \wedge \star u$, one obtains

$$
C \sum_{a=1}^{p}\left\|\vartheta u^{a}\right\|_{\tau}^{2} \leqslant\|\vartheta u\|_{\tau}^{2} \leqslant\left\|\vartheta_{\tau} u\right\|_{\tau}^{2}+\|\theta \wedge \star u\|_{\tau}^{2} \leqslant\left\|\vartheta_{\tau} u\right\|_{\tau}^{2}+C^{\prime}\|u\|_{\tau}^{2}
$$


Thus,

$$
\begin{aligned}
\left(1+C^{\prime}\right) \widetilde{D}_{\tau}^{2}(u) & \geqslant\|\bar{\partial} u\|_{\tau}^{2}+\left\|\vartheta_{\tau} u\right\|_{\tau}^{2}+C^{\prime}\|u\|_{\tau}^{2}+\|u\|_{\tau}^{2} \\
& \geqslant C \sum_{a=1}^{p}\left(\left\|\bar{\partial} u^{a}\right\|_{\tau}^{2}+\left\|\vartheta u^{a}\right\|_{\tau}^{2}+\left\|u^{a}\right\|_{\tau}^{2}\right)
\end{aligned}
$$

Thus,

$$
\left(1+C^{\prime}\right) \widetilde{D}_{\tau}^{2}(u) \geqslant C \sum_{a=1}^{p} D_{\tau}^{2}\left(u^{a}\right) .
$$

The inequalities (3.2) and (3.3) give the desired result.

Theorem 3.2. Let $X$ be an n-dimensional complex manifold and let $\Omega \Subset X$ be a bounded domain with $C^{2}$-smooth boundary satisfying condition $\left(H_{q}\right)$. Let $E \rightarrow X$ be a holomorphic vector bundle over $X$. For $q \geqslant 1$, there exists a bounded linear operator $N_{r, q}: L_{r, q}^{2}(\Omega, E) \rightarrow L_{r, q}^{2}(\Omega, E)$ such that

(i) Range $\left(N_{r, q}, E\right) \subset \operatorname{dom}\left(\square_{r, q}, E\right), N_{r, q} \square_{r, q}=I-\Pi_{r, q}$ on $\operatorname{dom}\left(\square_{r, q}, E\right)$;

(ii) for $u \in L_{r, q}^{2}(\Omega, E)$, we have $u=\bar{\partial} \bar{\partial}^{*} N_{r, q} u \oplus \bar{\partial}^{*} \bar{\partial} N_{r, q} u \oplus \Pi_{r, q} u$;

(iii) $N_{r, q}$ commutes with $\bar{\partial}$ and $\bar{\partial}^{*}, \Pi_{r, q} N_{r, q}=N_{r, q} \Pi_{r, q}=0$;

(iv) $N_{r, q}\left(C_{r, q}^{\infty}(\bar{\Omega}, E) \subset C_{r, q}^{\infty}(\bar{\Omega}, E)\right.$ and $\Pi_{r, q}\left(C_{r, q}^{\infty}(\bar{\Omega}, E)\right) \subset C_{r, q}^{\infty}(\bar{\Omega}, E)$.

Proof. Following Theorem 2.1, for $\tau=0$ and $u \in \operatorname{dom}\left(\square_{r, q}, E\right)$ of degree $q \geqslant 1$, we have $\|u\|^{2} \leqslant C\left\|\square_{r, q} u\right\|^{2}$. Since $\square_{r, q}$ is one to one on $\operatorname{dom}(\square, E)$ from $[9,(1.5 .3)]$, then there exists a unique bounded inverse operator $N_{r, q}$ : Range $\left(\square_{r, q}, E\right) \rightarrow \operatorname{dom}\left(\square_{r, q}, E\right) \cap$ $(\operatorname{ker}(\square, E))^{\perp}$ such that $N_{r, q} \square_{r, q} u=u$ on $\operatorname{dom}\left(\square_{r, q}, E\right)$. Thus, one can establish the existence theorem of the inverse of $\square_{r, q}$ the so called $\bar{\partial}$-Neumann operator $N_{r, q}$.

Theorem 3.3. Let $X$ be an $n$-dimensional complex manifold and let $\Omega \Subset X$ be a bounded domain with $C^{2}$-smooth boundary satisfying condition $\left(H_{q}\right)$. Let $E \rightarrow X$ be a holomorphic vector bundle over $X$. Then, for $f \in L_{r, q}^{2}(X, E), \operatorname{supp} f \subset \bar{\Omega}, 1 \leqslant q \leqslant$ $n-1$, satisfying $\bar{\partial} f=0$ in the distribution sense in $X$, there exists $u \in L_{r, q-1}^{2}(X, E)$, $\operatorname{supp} u \subset \bar{\Omega}$ such that $\bar{\partial} u=f$ in the distribution sense in $X$.

Proof. Let $f \in L_{r, q}^{2}(X, E)$, supp $f \subset \bar{\Omega}$, then $f \in L_{r, q}^{2}(\Omega, E)$. From Theorem 2.2, $N_{n-r, n-q}$ exists for $n-q \geqslant 1$. Since $N_{n-r, n-q}=\left(\square_{n-r, n-q}\right)^{-1}$ on Range $\left(\square_{n-r, n-q}, E^{*}\right)$ and Range $\left(N_{n-r, n-q}, E^{*}\right) \subset \operatorname{dom}\left(\square_{n-r, n-q}, E^{*}\right)$, then

$$
N_{n-r, n-q} \#_{E} \star f \in \operatorname{dom}\left(\square_{n-r, n-q}, E^{*}\right) \subset L_{n-r, n-q}^{2}\left(\Omega, E^{*}\right),
$$

for $q \leqslant n-1$. Thus, we can define $u \in L_{r, q-1}^{2}(\Omega, E)$ by

$$
u=-\star \#_{E^{*}} \bar{\partial} N_{n-r, n-q} \#_{E} \star f .
$$

Now, we extend $u$ to $X$ by defining $u=0$ in $X \backslash \bar{\Omega}$. As in [1], the extended form $u$ satisfies the equation $\bar{\partial} u=f$ in the distribution sense in $X$. 


\section{The $L^{2} \bar{\partial}$ Cauchy Problem on Piecewise Smooth Strongly PSEudoconvex Domains}

A relatively compact open subset $\Omega$ of $X$ has piecewise strongly pseudoconvex boundary $b \Omega$, if $b \Omega$ is covered by finitely many open subsets $\left\{U_{j}\right\}, 1 \leqslant j \leqslant k$, of $X$ and there are $C^{2}$ strictly plurisubharmonic functions $\delta_{j}$ on $\left\{U_{j}\right\}, 1 \leqslant j \leqslant k$, such that $\Omega \cap\left(\bigcup_{j=1}^{k} U_{j}\right)$ is the set of all $x \in \bigcup_{j=1}^{k} U_{j}$ which, for every $1 \leqslant j \leqslant k$, satisfy $x \notin U_{j}$ or $\delta_{j}(x)<0$.

The boundary $b \Omega$ need not be piecewise smooth, so we do not require any further conditions on the $\delta_{j}, 1 \leqslant j \leqslant k$. Following P. W. Darko [7], one have the following result.

Theorem 4.1. Let $X$ be an $n$-dimensional complex manifold with a $C^{\infty}$ Hermitian metric. Let $\Omega \Subset X$ be a strongly pseudoconvex domain with piecewise smooth boundary. For $f \in L_{r, q}^{2}(X), 1 \leqslant q \leqslant n-1$, satisfying $\bar{\partial} f=0$ in the distribution sense in $X$, there exists $u \in L_{r, q-1}^{2}(X)$, such that $\bar{\partial} u=f$ in the of distributions and $\|u\|^{2} \leqslant C\|f\|^{2}$, where $C$ depends on $\Omega$ and $r, q$ but not on $f$.

Proposition 4.1. Let $X$ and $\Omega$ be the same as in Theorem 3.1. Then, there exists a uniquely bounded linear operator $N_{r, q}: L_{r, q}^{2}(\Omega) \rightarrow L_{r, q}^{2}(\Omega)$, such that $\square_{r, q} \circ N_{r, q} u=u$ for any $u \in L_{r, q}^{2}(\Omega)$.

Proof. Following Theorem 3.1 as in [5, Section 4.4], we have $\mathcal{H}_{r, q}(\Omega)=\{0\}$ for $q>0$ and for every $f \in L_{r, q}^{2}(\Omega), 1 \leqslant q \leqslant n-1$, there exists $u \in \operatorname{dom} \bar{\partial} \cap \operatorname{dom} \bar{\partial}^{*}$ with $\bar{\partial}^{*} u \in \operatorname{dom} \bar{\partial}$ and $\bar{\partial} u \in \operatorname{dom} \bar{\partial}^{*}$, such that

$$
\bar{\partial}^{*} u+\bar{\partial}^{*} \bar{\partial} u=f \text { and }\|u\|^{2} \leqslant C\|f\|^{2},
$$

where $C$ depends on $\Omega$ and $r, q$ but not on $f$. Theorem 3.1 implies the fundamental estimate

$$
\|u\|^{2} \leqslant C\left(\|\bar{\partial} u\|^{2}+\left\|\bar{\partial}^{*} u\right\|^{2}\right) .
$$

Then there exists a uniquely determined bounded linear operator $N_{r, q}: L_{r, q}^{2}(\Omega) \rightarrow$ $L_{r, q}^{2}(\Omega)$, such that $\square_{r, q} \circ N_{r, q} u=u$ for any $u \in L_{r, q}^{2}(\Omega)$.

Theorem 4.2. Let $X$ and $\Omega$ be the same as in Theorem 3.1. For $f \in L_{r, q}^{2}(X)$, $1 \leqslant q \leqslant n-1$, supp $f \subset \bar{\Omega}$, satisfying $\bar{\partial} f=0$ in the distribution sense in $X$, there exists $u \in L_{r, q-1}^{2}(X), \operatorname{supp} u \subset \bar{\Omega}$ such that $\bar{\partial} u=f$ in the distribution sense in $X$.

Proof. Let $f \in L_{r, q}^{2}(X)$, supp $f \subset \bar{\Omega}$, then $f \in L_{r, q}^{2}(\Omega)$. Since $N_{n-r, n-q}$ exists for $n-q \geqslant 1$. By defining $u$ as in (2.4), then $\operatorname{supp} u \subset \bar{\Omega}$ and $u$ vanishes on $b \Omega$. As in Saber [20], one can prove that the extended form $u$ satisfies the equation $\bar{\partial} u=f$ in the distribution sense in $X$.

\section{The Weighted $L^{2} \bar{\partial}$ Cauchy Problem}

Let $\left(x_{0}, x_{1}, \ldots, x_{n}\right)$ be a (fixed) homogeneous coordinates of $\mathbb{P}^{n}$ and let $\omega$ be the Fubini-Study metric of the complex projective space of $\mathbb{P}^{n}$ determined by 
$\left(x_{0}, x_{1}, \ldots, x_{n}\right)$. If, for example, $U_{0}$ is the open set in $\mathbb{P}^{n}$ defined by $x_{0} \neq 0$ and if $\left(z_{1}, z_{2}, \ldots, z_{n}\right)$, where $z_{i}=x_{i} / x_{0}$ is the homogeneous coordinates of $U_{0}$, then the metric $\omega$ is written in the form

$$
\omega=\frac{\sum_{i=1}^{n}\left|d z_{i}\right|^{2}}{1+\sum_{i=1}^{n}\left|z_{i}\right|^{2}}-\frac{\left|\sum_{i=1}^{n} z_{i} d \bar{z}_{i}\right|^{2}}{\left(1+\sum_{i=1}^{n}\left|z_{i}\right|^{2}\right)^{2}}, \quad \text { on } U_{0} .
$$

This is well-known standard Kähler metric of $\mathbb{P}^{n}$. Let $\nabla$ be the Levi-Civita connection of $\mathbb{P}^{n}$ with the standard Fubini-Study metric $\omega$. The Levi-Civita connection, sometimes also known as the Riemannian connection or covariant derivative. Let $\left\{e_{i}\right\}$ be an orthonormal basis of vector fields. For any two vector fields $u, v$, the curvature operator of the connection $\nabla$ is given by $\mathcal{R}(u, v)=\nabla_{u} \nabla_{v}-\nabla_{v} \nabla_{u}-\nabla_{[u, v]}$. with $\mathcal{R}_{i j k l}=g\left(\mathcal{R}\left(e_{i}, e_{j}\right) e_{k}, e_{l}\right)$. We also define the Ricci tensor $\mathcal{R}_{i j}=\sum_{k} \varepsilon_{k} \mathcal{R}_{i k k j}$ which turns out to be self-adjoint with respect to $\omega$ and the scalar curvature $\Theta$ as the trace of the Ricci tensor

$$
\Theta=\sum_{i} \mathcal{R}_{i i}=\sum_{i, j} \varepsilon_{i} \varepsilon_{j} \mathcal{R}_{j i i j}
$$

Let $\operatorname{dist}(z, b \Omega)$ be the Fubini distance from $z \in \Omega$ to the boundary $b \Omega$ and let $\delta: \mathbb{P}^{n} \rightarrow \mathbb{R}$ be a $C^{2}$ defining function for $\Omega$ normalized by $|d \delta|=1$ on $b \Omega$ such that

$$
\delta=\delta(z)= \begin{cases}l l-\operatorname{dist}(z, b \Omega), & \text { if } z \in \Omega, \\ \operatorname{dist}(z, b \Omega), & \text { if } z \in \mathbb{P}^{n} \backslash \Omega\end{cases}
$$

where $\delta$ is computed with respect to the Kähler metric $\omega$ on $\mathbb{P}^{n}$.

Proposition 5.1 (Bochner-Hörmander-Kohn-Morrey formula). Let $\Omega$ be a bounded domain with $C^{2}$-smooth boundary $b \Omega$ and $C^{2}$-defining function $\delta(z)$. Then, for any $u \in C_{r, q}^{\infty}(\bar{\Omega}) \cap \operatorname{dom} \bar{\partial}_{\phi}^{*}$, with $1 \leqslant q \leqslant n-1$ and $\phi \in C^{2}(\bar{\Omega})$, we have

$$
\begin{aligned}
\|\bar{\partial} u\|_{\phi}^{2}+\left\|\bar{\partial}_{\phi}^{*} u\right\|_{\phi}^{2}= & \langle\Theta u, \bar{u}\rangle_{\phi}+\sum_{I, J} \sum_{k=1}^{n} \int_{\Omega}\left|\frac{\partial u_{I, J}}{\partial \bar{z}^{k}}\right|^{2} e^{-\phi} d V+\langle(i \partial \bar{\partial} \phi) u, \bar{u}\rangle_{\phi} \\
& +\sum_{I, K} \sum_{\alpha, \beta=1}^{n} \int_{b \Omega} \frac{\partial^{2} \delta}{\partial z^{\alpha} \partial \bar{z}^{\beta}} u_{I, \alpha K} \bar{u}_{I, \beta K} e^{-\phi} d S
\end{aligned}
$$

Proof. This formula is known (cf. [9]) for some special cases. For the case $\phi=0$, the stated formula was proved in Siu [22].

Proposition 5.2. ([17, Corollary 6.5]). Let $\Omega \Subset \mathbb{P}^{n}$ be a bounded domain with $C^{2}$ smooth boundary satisfying condition $\left(H_{q}\right)$. Then, the Levi form of the function $\delta$ has at least $n-q+1$ positive eigenvalues at each point of $\Omega$. 
Proposition 5.3. Suppose that $\Theta$ is the curvature term defined in (5.1) with respect to the Fubini-Study metric $\omega$. Then, for any $(r, q)$-form $u$ of $\Omega \Subset \mathbb{P}^{n}$ with $q \geqslant 1$,

$$
\begin{aligned}
& (\Theta u, \bar{u})=q(2 n+1)|u|^{2}, \quad \text { when } u \text { is } a(0, q) \text {-form, } \\
& (\Theta u, \bar{u})=0, \quad \text { for any }(n, q) \text {-form } u, \\
& (\Theta u, \bar{u}) \geqslant 0, \quad \text { when } r \geqslant 1 \text { andu is } a(r, q) \text {-form. }
\end{aligned}
$$

In fact, the assertion for $(0, q)$-forms and $(n, q)$-forms was computed in [23]. Also, following Lemma 3.3 of Henkin-Iordan [8] and its proof showed that the curvature operator $\Theta$ acting on $L_{r, q}^{2}(\Omega)$ is a semi-positive operator.

Theorem 5.1. Let $\Omega \Subset \mathbb{P}^{n}$ be a bounded domain with $C^{2}$-smooth boundary satisfying condition $\left(H_{q}\right)$. For any $1 \leqslant q \leqslant n$ and $t>0$, there exists a bounded linear operator $N_{r, q}^{t}: L_{r, q}^{2}(\Omega) \rightarrow L_{r, q}^{2}(\Omega)$ satisfies the following properties:

(i) Range $\left(N_{r, q}^{t}\right) \subset \operatorname{dom}\left(\square_{r, q}^{t}\right), N_{r, q}^{t} \square_{r, q}^{t}=I$ on $\operatorname{dom}\left(\square_{r, q}^{t}\right)$;

(ii) for $f \in L_{r, q}^{2}(\Omega)$, we have $u=\bar{\partial} \bar{\partial}_{t}^{*} N_{r, q}^{t} f \oplus \bar{\partial}_{t}^{*} \bar{\partial} N_{r, q}^{t} f$,

(iii) $\bar{\partial} N_{r, q}^{t}=N_{r, q+1}^{t} \bar{\partial}, 1 \leqslant q \leqslant n-1$ and $\bar{\partial}_{t}^{*} N_{r, q}^{t}=N_{r, q-1}^{t} \bar{\partial}_{t}^{*}, 2 \leqslant q \leqslant n$,

(iv) $N_{r, q}^{t}, \bar{\partial} N_{r, q}^{t}$ and $\bar{\partial}_{t}^{*} N_{r, q}^{t}$ are bounded operators with respect to the $L^{2}$-norms.

Proof. By choosing $\phi_{t}=-t \log |\delta|, t>0$ in Proposition 4.1, and using Proposition 4.2 and Proposition 4.3, the identity (5.2) implies the weighted $L^{2}$-existence for the $\bar{\partial}$. Also, for $u \in \operatorname{dom}\left(\square_{r, q}^{t}\right)$ of degree $q \geqslant 1$, we have for $t>0, t\|u\|_{t} \leqslant\left\|\square_{r, q}^{t} u\right\|_{t}$. Then, as in Theorem 2.2 there exists a unique bounded inverse operator $N_{r, q}^{t}$ : Range $\left(\square_{r, q}^{t}\right) \rightarrow$ $\operatorname{dom}\left(\square_{r, q}^{t}\right) \cap\left(\operatorname{ker}\left(\square_{r, q}^{t}\right)\right)^{\perp}$, such that $N_{r, q}^{t} \square_{r, q}^{t} f=f$ on dom $\left(\square_{r, q}^{t}\right)$. Therefore, one can establish the existence theorem of the inverse of $\square_{r, q}^{t}$ the so called weighted $\bar{\partial}$-Neumann operator $N_{r, q}^{t}$.

Theorem 5.2. Let $\Omega \Subset \mathbb{P}^{n}$ be a bounded domain with $C^{2}$-smooth boundary satisfying condition $\left(H_{q}\right)$. Then, for $f \in L_{r, q}^{2}\left(\delta^{-t}\right), 1 \leqslant q \leqslant n-1$, satisfying $\bar{\partial} f=0$ in the distribution sense in $\mathbb{P}^{n}$ and $f$ is supported in $\bar{\Omega}$, there exists $u \in L_{r, q-1}^{2}\left(\delta^{-t}\right)$ such that $\bar{\partial} u=f$ in the distribution sense in $\mathbb{P}^{n}$ with $u$ is supported in $\bar{\Omega}$ and

$$
\int_{\Omega}|u|^{2} \delta^{-t} d V \leq C \int_{\Omega}|f|^{2} \delta^{-t} d V
$$

for some $C>0$.

Proof. Following Theorem 4.1, $N_{r, q}^{t}$ exists for forms in $L_{n-r, n-q}^{2}\left(\mathbb{P}^{n}, \delta^{t}\right)$. Let $\star_{t}$ denote the weighted Hodge-star operator with respect to the weighted norm of $L_{r, q}^{2}\left(\mathbb{P}^{n}, \delta^{t}\right)$. Then $\star_{t}=\delta^{t} \star=\star \delta^{t}$, where $\star$ is the Hodge star operator with the unweighted $L^{2}$ norm. Thus, we can define $u \in L_{r, q-1}^{2}\left(\mathbb{P}^{n}, \delta^{-t}\right)$ by

$$
u=-\star_{t} \overline{\bar{\partial}} N_{n-r, n-q} \star_{-t} \bar{f} .
$$

Thus supp $u \subset \bar{\Omega}$ and $u$ vanishes on $b \Omega$. Now, we extend $u$ to $\mathbb{P}^{n}$ by defining $u=0$ in $\mathbb{P}^{n} \backslash \Omega$. As in Saber [20], the extended form $u$ satisfies the equation $\bar{\partial} u=f$ in the distribution sense in $\mathbb{P}^{n}$. 
The above result can be extended easily to the following result.

Theorem 5.3. Let $X$ be an $n$-dimensional Kähler manifold with semi-positive holomorphic bisectional curvature and let $\Omega \Subset X$ be a bounded domain with $C^{2}$-smooth boundary satisfying condition $\left(H_{q}\right)$. Then, for $f \in L_{r, q}^{2}\left(\delta^{-t}\right), 1 \leqslant q \leqslant n-1$, satisfying $\bar{\partial} f=0$ in the distribution sense in $X$ and $f$ is supported in $\bar{\Omega}$, there exists $u \in L_{r, q-1}^{2}\left(\delta^{-t}\right)$ such that $\bar{\partial} u=f$ in the distribution sense in $X$ with $u$ is supported in $\bar{\Omega}$ and

for some $C>0$.

$$
\int_{\Omega}|u|^{2} \delta^{-t} d V \leq C \int_{\Omega}|f|^{2} \delta^{-t} d V
$$

\section{Global Boundary Regularity for $\bar{\partial}_{\mathrm{Mix}}$}

In this section, we study the global regularity for the $\bar{\partial}_{m i x}$-equation with mixed boundary conditions. Throughout this section, $\Omega$ will denote the annulus in a Stein manifold $X$ between $\Omega_{1}$ and $\Omega_{2}$ with $C^{3}$ boundary. Let $\delta \in C^{3}\left(\bar{\Omega}_{2}\right)$ be the defining function of $\Omega$. We impose the $\bar{\partial}$-Dirichlet boundary condition on $\Omega_{1}$ and the $\bar{\partial}$ Neumann boundary condition on $\Omega_{2}$. We say that $U$ satisfies the $\bar{\partial}$-Dirichlet condition along $\Omega_{2}$ if $\left.U_{J}\right|_{\Omega_{2}} \equiv 0$ whenever $J=\left(j_{1}, \ldots, j_{q}\right)$ with $j_{q} \neq n$. We say $U$ satisfies the $\bar{\partial}$-Neumann condition along $\Omega_{1}$ if $\left.U_{J}\right|_{\Omega_{1}} \equiv 0$ when $j_{q}=n$.

Definition 6.1. For $0 \leqslant r \leqslant n, 0 \leqslant q \leqslant n$ and $u \in L_{r, q}^{2}(\Omega), u \in \operatorname{dom} \bar{\partial}_{\text {mix }}$ if and only if there exists $f \in L_{r, q+1}^{2}(\Omega)$ and a sequence $\left\{u_{\nu}\right\} \in L_{r, q}^{2}(\Omega)$ which vanish near $b \Omega_{2}$ such that $u_{\nu} \rightarrow u$ in $L_{r, q}^{2}(\Omega)$ and $\bar{\partial} u_{\nu} \rightarrow u$ in $L_{r, q+1}^{2}(\Omega)$, then we say $u \in \operatorname{dom} \bar{\partial}_{\text {mix }}$ and $\bar{\partial}_{\text {mix }} u=f$.

Let $\bar{\partial}_{t, \text { mix }}^{*}$ be the Hilbert-space adjoint of $\bar{\partial}_{\text {mix }}$. Let $B_{r, q}^{2}(\Omega)$ denote the space of $(r, q)$-forms which are $C^{2}$-smooth in a neighborhood of $\bar{\Omega}$ and satisfies $\bar{\partial}$-Dirichlet condition on $b \Omega_{2}$ and $\bar{\partial}$-Neumann condition on $b \Omega_{1}$. Denote by $W_{r, q}^{m}(\Omega), m \in \mathbb{R}$, the Hilbert spaces of $(r, q)$-forms with $W^{m}(\Omega)$-coefficients and their norms are denoted by $\|u\|_{W^{m}}$.

As in Lemma 6.4 of [3] and Lemma 4.3.2 in [5], the Hörmander-Friderichs smooth lemma also holds in this setting: Let $u \in \operatorname{dom} \bar{\partial}_{\text {mix }} \cap \operatorname{dom} \bar{\partial}_{\text {mix }}^{*} \cap L_{r, q}^{2}(\Omega, \phi)$, there exists $\left\{u_{\nu}\right\} \in L_{r, q}^{2}(\Omega, \phi)$ such that

$$
\left\|u_{\nu}-u\right\|+\left\|\bar{\partial}_{\text {mix }} u_{\nu}-\bar{\partial}_{\text {mix }} u\right\|+\left\|\bar{\partial}_{\text {mix }}^{*} u_{\nu}-\bar{\partial}_{\text {mix }}^{*} u\right\| \rightarrow 0
$$

From now on we fix $\phi_{t}(z)=t|z|^{2}$ near $b \Omega_{1}$ and $\phi_{t}(z)=t\left(|z|^{2}-\tau \delta\right)$ near $b \Omega_{2}$, where $t$ and $\tau$ are positive constants which will be determined later. Let $\square_{\text {mix }}^{t}=$ $\bar{\partial}_{\text {mix }} \bar{\partial}_{t, \text { mix }}^{\star}+\bar{\partial}_{t, \text { mix }}^{\star} \bar{\partial}_{\text {mix }}$ be the complex Laplacian operator and take $f \in \operatorname{dom}\left(\square_{\text {mix }}^{t}\right)$ of degree $q \geqslant 1$, then we have for every $t>0$.

The proof of the following proposition follows by using a partition of unity.

Proposition 6.1. Let $\Omega=\Omega_{1} \backslash \bar{\Omega}_{2}$ be an annulus domain in a Stein manifold $X$ between an internal domain $\Omega_{2}$ satisfies condition $\left(H_{n-q-1}\right)$ and an external domain 
$\Omega_{1}$ satisfies condition $\left(H_{q}\right)$. There exist a positive constant $t_{*}$ such that for any $t \geqslant t_{*}$, the harmonic space $\mathcal{H}_{r, q}^{m}(E)$ has finite dimension and there exists a positive constant $C_{t}$ depending on $t$ such that

$$
\|u\|_{\phi_{t}}^{2} \leqslant C_{t}\left(\left\|\bar{\partial}_{m i x} u\right\|_{\phi_{t}}^{2}+\left\|\bar{\partial}_{t, m i x}^{*} u\right\|_{t}^{2}\right),
$$

for $u \in \operatorname{dom}\left(\bar{\partial}_{m i x}\right) \cap \operatorname{dom}\left(\bar{\partial}_{t, m i x}^{*}\right)$ with $q \geqslant 1$.

Proof. Assume first $u$ is supported in a small neighborhood $U$ of $p \in b \Omega$. Since $u$ satisfies $\bar{\partial}$-Dirichlet condition on $b \Omega_{2}$, then $\star_{t} u$ satisfies $\bar{\partial}$-Neumann condition on $b \Omega_{2}$. Now $1 \leqslant n-q \leqslant n-2$, since $2 \leqslant q \leqslant n-1$. Thus, for $u \in B_{r, q}^{2}(\Omega)$, by a similar argument of Proposition 3.1 in Saber [19], we have

$$
t\left\|\star_{t} u\right\|_{-t}^{2} \leqslant\left\|\bar{\partial} \star_{t} u\right\|_{-t}^{2}+\left\|\bar{\partial}^{*} \star_{t} u\right\|_{-t}^{2},
$$

when $t$ is sufficiently large. Since the Hodge star operator $\star$ is an isometry operator in $L^{2}$-space, we have

$$
t\left\|\star_{t} u\right\|_{-t}^{2} \leqslant\left\|\star_{t} \bar{\partial} \star_{t} u\right\|_{t}^{2}+\left\|\star_{t} \bar{\partial}^{*} \star_{t} u\right\|_{t}^{2} .
$$

Substituting the identity $\bar{\partial}_{\text {mix }}=\star_{t} \bar{\partial}^{*} \star_{t}$ and $\bar{\partial}_{t, \text { mix }}^{*}=\star_{t} \bar{\partial} \star_{t}$ to (6.3). It follows that

$$
t\|u\|_{t}^{2} \leqslant\left\|\bar{\partial}_{t, \text { mix }}^{*} u\right\|_{t}^{2}+\left\|\bar{\partial}_{\text {mix }} u\right\|_{t}^{2},
$$

for all $u \in B_{r, q}^{2}(\Omega)$. Then (6.1) shows that (6.2) holds for all $u \in \operatorname{dom} \bar{\partial}_{\text {mix }} \cap$ $\operatorname{dom} \bar{\partial}_{t, \text { mix }}^{*} \cap L_{r, q}^{2}\left(\Omega, \phi_{t}\right)$.

Theorem 6.1. Let $X$ and $\Omega$ be the same as in Proposition 5.1. There exists a positive integer $t^{*}$ such that, for $t \geqslant t^{*}, r \geqslant 0, q \geqslant 1$, there exists a bounded linear operator $N_{\text {mix }}^{t}: L_{r, q}^{2}(\Omega) \rightarrow L_{r, q}^{2}(\Omega)$ such that

(i) Range $\left(N_{\text {mix }}^{t}\right) \subset \operatorname{dom}\left(\square_{\text {mix }}^{t}\right), N_{\text {mix }}^{t} \square_{\text {mix }}^{t}=I-\mathbb{H}_{\text {mix }}$ on $\operatorname{dom}\left(\square_{\text {mix }}^{t}\right)$;

(ii) for $u \in L_{r, q}^{2}(\Omega)$, we have $u=\bar{\partial}_{\text {mix }} \bar{\partial}_{t, \text { mix }}^{*} N_{\text {mix }}^{t} u \oplus \bar{\partial}_{t, \text { mix }}^{*} \bar{\partial}_{\text {mix }} N_{\text {mix }}^{t} u \oplus \mathbb{H}_{\text {mix }} u$;

(iii) $N_{m i x}^{t} \bar{\partial}_{m i x}=\bar{\partial}_{m i x} N_{m i x}^{t}$ on $\operatorname{dom}\left(\bar{\partial}_{m i x}\right)$;

(iv) $N_{m i x}^{t} \bar{\partial}_{t, m i x}^{*}=\bar{\partial}_{t, m i x}^{*} N_{m i x}^{t}$ on $\operatorname{dom}\left(\bar{\partial}_{t, m i x}^{*}\right)$;

(v) $N_{\text {mix }}^{t}, \bar{\partial}_{\text {mix }} N_{\text {mix }}^{t}$ and $\bar{\partial}_{t, \text { mix }}^{*} N_{\text {mix }}^{t}$ are bounded operators with respect to the $L^{2}$ norms.

Proof. Following Proposition 5.1, one obtain that

$$
t\|f\|_{t} \leq\left\|\square_{\text {mix }}^{t} f\right\|_{t} .
$$

Since $\square_{\text {mix }}^{t}$ is a linear closed densely defined operator, then, from [9, Theorem 1.1.1], Range $\left(\square_{\text {mix }}^{t}\right)$ is closed. Thus, from (1.1.1) in [9] and the fact that $\square_{r, q}$ is self adjoint, we have the Hodge decomposition

$$
L_{r, q}^{2}(\Omega)=\bar{\partial}_{\text {mix }} \bar{\partial}_{t, \text { mix }}^{\star} \text { dom } \square_{\text {mix }}^{t} \oplus \bar{\partial}_{t, \text { mix }}^{\star} \bar{\partial}_{\text {mix }} \text { dom } \square_{\text {mix }}^{t}
$$

Since $\square_{\text {mix }}^{t}$ is one to one on dom $\square_{\text {mix }}^{t}$ from (1.5.3) in [9], then there exists a unique bounded inverse operator $N_{\text {mix }}^{t}$ : Range $\square_{\text {mix }}^{t} \rightarrow \operatorname{dom} \square_{\text {mix }}^{t} \cap\left(\operatorname{ker} \square_{\text {mix }}^{t}\right)^{\perp}$ such that $N_{\text {mix }}^{t} \square_{\text {mix }}^{t} f=f$ on dom $\square_{\text {mix }}^{t}$. Thus, we can establish the existence theorem of the inverse of $\square_{\text {mix }}^{t}$ the so called weighted $\bar{\partial}$-Neumann operator $N_{\text {mix }}^{t}$. 
Corollary 6.1. (i) If $f \in \operatorname{ker} \bar{\partial}_{\text {mix }}$, then $\bar{\partial}_{t, m i x}^{*} N_{\text {mix }}^{t} f$ gives the solution $u_{t}$ to the equation $\bar{\partial}_{m i x} u_{t}=f$ of minimal $u_{t} \in L_{r, q-1}^{2}(\Omega)$-norm.

(ii) If $f \in \operatorname{ker} \bar{\partial}_{t, m i x}^{*}$, then $\bar{\partial}_{m i x} N_{m i x}^{t} f$ gives the solution $u_{t}$ to the equation $\bar{\partial}_{t, m i x}^{*} u_{t}=$ $f$ of minimal $u_{t} \in L_{r, q+1}^{2}(\Omega)$-norm.

Using the elliptic regularization method which was used in [14], one can pass from the a priori estimates (6.4) to actual estimates and we can prove the following theorem.

Theorem 6.2. For every integer $T \geqslant 0$ and real $t>T>0, N_{\text {mix }}^{t}$ is bounded from $W_{r, q}^{m}(\Omega)$ into itself.

By Theorem $5.1(v)$, Theorem 5.2 and the density of $C_{r, q}^{\infty}(\bar{\Omega})$ in $W_{r, q}^{m}(\Omega)$, the following is immediate.

Corollary 6.2. If $f \in W_{r, q}^{m}(\Omega), m=0,1,2,3, \ldots$ satisfies $\bar{\partial}_{\text {mix }} f=0$, where $q \geqslant 1$, then there exits $u \in W_{r, q-1}^{m}(\Omega)$ so that $\bar{\partial}_{\text {mix }} u=f$ on $\Omega$ with estimate $\|u\|_{W^{m}} \leq$ $C_{m}\|f\|_{W^{m}}$.

Theorem 6.3. For $f \in C_{r, q}^{\infty}(\bar{\Omega})$, with $\bar{\partial}_{\text {mix }} f=0, q \geqslant 1$, there exists $u \in C_{r, q-1}^{\infty}(\bar{\Omega})$ such that $\bar{\partial}_{\text {mix }} u=f$.

Proof. From Corollary 5.1, there is $u_{k} \in W_{r, q-1}^{k}(\Omega)$ satisfying $\bar{\partial}_{\text {mix }} u_{k}=f$ for each positive integer $k$. We shall modify $u_{k}$ to generate a new sequence that converges to a smooth solution. Since $u_{k}-u_{k+1}$ is in $W_{r, q-1}^{k}(\Omega) \cap \operatorname{ker}\left(\bar{\partial}_{\text {mix }}\right)$, there exists a $v_{k+1} \in W_{r, q-1}^{k+1}(\Omega) \cap \operatorname{ker}\left(\bar{\partial}_{\text {mix }}\right)$ such that

$$
\left\|u_{k}-u_{k+1}-v_{k+1}\right\|_{W^{k}} \leq 2^{-k}, \quad k=1,2,3, \ldots
$$

Setting $\widetilde{u}_{k+1}=u_{k+1}+v_{k+1}$, then $\widetilde{u}_{k+1} \in W_{r, q-1}^{k+1}(\Omega)$ and $\bar{\partial}_{\text {mix }} \widetilde{u}_{k}=f$. Inductively, we can choose a new sequence $\widetilde{u}_{k} \in W_{r, q-1}^{k}(\Omega)$ such that $\bar{\partial}_{\text {mix }} \widetilde{u}_{k}=f$ and

$$
\left\|\widetilde{u}_{k+1}-\widetilde{u}_{k}\right\|_{W^{k}} \leq 2^{-k}, \quad k=1,2,3, \ldots
$$

Set $u_{\infty}=\widetilde{u}_{t}+\sum_{k=t}^{\infty}\left(\widetilde{u}_{k+1}-\widetilde{u}_{k}\right), t \in \mathbb{N}$. Then $u_{\infty}$ is well defined and is in $W_{r, q-1}^{k}(\Omega)$ for every $k$. Thus, by the Sobolev embedding theorem, $u_{\infty} \in C_{r, q}^{\infty}(\bar{\Omega})$ and $\bar{\partial}_{\text {mix }} u_{\infty}=f$. Thus the proof follows.

Corollary 6.3. We assume that $0 \leqslant r \leqslant n, 2 \leqslant q \leqslant n$ and the boundary of $\Omega$ is smooth. Let $N_{m i x}^{t}$ be the weighted $\bar{\partial}_{\text {mix }}$-Neumann operator. For every $k \geqslant$ 0 , there exists $S_{k}$ such that when $t \geqslant S_{k}$ we have that $N_{m i x}^{t}, \bar{\partial}_{m i x} N_{m i x}^{t}, \bar{\partial}_{t, m i x}^{*} N_{m i x}^{t}$, $\bar{\partial}_{t, m i x}^{*} N_{m i x}^{t} \bar{\partial}_{m i x}$ are exactly regular on $W_{r, q}^{k}(\Omega)$.

Proof. When $f \in C_{r, q}^{\infty}(\bar{\Omega}) \cap \operatorname{dom} \bar{\partial}_{\text {mix }}$ and $\operatorname{supp} f \Subset U \cap \bar{\Omega}$, where $U$ is a special boundary chart, then from (6.4), we have that

$$
t\|f\|_{t}^{2} \leqslant C^{2}\left\|\square_{\text {mix }}^{t} f\right\|_{t}^{2} .
$$


When $f \in C_{r, q}^{\infty}(\Omega)$ with supp $f$ a compact subset in $\Omega$, we have the following Garding's inequality

$$
\|f\|_{W^{1}}^{2} \leqslant\left\|\bar{\partial}_{\text {mix }} f\right\|_{t}^{2}+\left\|\bar{\partial}_{t, \text { mix }}^{*} f\right\|_{t}^{2}+C_{t}\|f\|_{t}^{2} .
$$

Combining (6.5) and (6.6) and with a similar argument as in Kohn [14], the result follows.

Corollary 6.4. Suppose that $f \in L_{r, q}^{2}(\Omega) \cap \operatorname{ker} \bar{\partial}_{\text {mix }}$, where $2 \leqslant q \leqslant n-1$. Then for each $k>0$, there exists $f_{n} \in W_{r, q}^{k}(\Omega)$ with $f_{n}$ satisfying $\bar{\partial}$-Dirichlet condition on $b \Omega_{2}$ such that $f_{n} \rightarrow f$ in $L_{r, q}^{2}(\Omega)$ and $\bar{\partial}_{\text {mix }} f_{n}=0$.

Corollary 6.5. Suppose that $f \in C_{r, q}^{\infty}(\bar{\Omega}) \cap \operatorname{ker} \bar{\partial}_{\text {mix }}$, where $0 \leqslant p \leqslant n, 2 \leqslant q \leqslant n-1$. Then, there exists $u \in C_{r, q-1}^{\infty}(\bar{\Omega}) \cap$ dom $\bar{\partial}_{\text {mix }}$ satisfying $\bar{\partial}$-Dirichlet condition on $b \Omega_{2}$ such that $f_{n} \longrightarrow f$ in $L_{r, q}^{2}(\Omega)$ and $\bar{\partial}_{m i x} u=f$.

\section{7. $L^{2}$ THEORY FOR $\bar{\partial}_{\text {MIX }}$}

We consider an operator $\bar{\partial}_{\text {mix }}$ which satisfies that $\bar{\partial}_{c} \subseteq \bar{\partial}_{\text {mix }} \subseteq \bar{\partial}$, where $\bar{\partial}$ is the maximal realization of the differential operator $\bar{\partial}$. As Theorem 2.2 in Li-Shaw [16], we prove the following theorem.

Theorem 7.1. Let $X$ and $\Omega$ be the same as in Proposition 5.1. Then, for $0 \leqslant r \leqslant n$, $2 \leqslant q \leqslant n$, the Dolbeault cohomology $H_{L^{2}}^{r, q}(\Omega)$ with $L^{2}(\Omega)$-coefficients vanishes, i.e.,

$$
H_{\bar{\partial}_{m i x}^{r, L^{2}}}^{r, q}(\Omega)=\frac{\left\{f \in L_{r, q}^{2}(\Omega): \bar{\partial}_{\text {mix }} f=0\right\}}{\left\{f \in L_{r, q}^{2}(\Omega): f=\bar{\partial}_{\text {mix }} u, u \in L_{r, q-1}^{2}(\Omega)\right\}}=\{0\} .
$$

Proof. Let $f \in L_{r, q}^{2}(\Omega)$ with $\bar{\partial}_{\text {mix }} f=0$. Extending $f$ to be zero in $\Omega_{2}$, denoted by $f^{0}$, we have that $f^{0} \in L_{r, q}^{2}\left(\Omega_{1}\right)$ and $\bar{\partial} f^{0}=0$ in $\Omega_{1}$. This follows from the assumption that $\Omega_{2}$ has $C^{2}$ boundary and the strong $\bar{\partial}_{c}$ and weak $\bar{\partial}_{c}$ are equal. Here we only need the boundary $\Omega_{2}$ to be Lipschitz. For a proof of such weak equal strong results, see e.g. Lemma 2.4 in Laurent-Thiébaut-Shaw [15]. Thus we have from the $L^{2}$ theory for bounded domains satisfies condition $\left(H_{q}\right)$, there exists a solution $v \in L_{r, q-1}^{2}\left(\Omega_{1}\right)$ such that $\bar{\partial} v=f^{0}$ in $\Omega_{1}$. From the elliptic regularity in the interior for $\bar{\partial}$, we can assume that the form $v$ is in $W_{r, q-1}^{1}\left(\Omega_{2}\right)$. The form $v$ satisfies $\bar{\partial} v=0$ on $\Omega_{2}$. Since $q>1$ and the boundary of $\Omega_{2}$ is $C^{2}$-smooth, there exists a solution $w \in W_{r, q-1}^{1}\left(\Omega_{2}\right)$ such that $\bar{\partial} w=v$ in $\Omega_{2}$. This follows from a result of Ho [10] for sufficiently smooth boundary when the boundary is only $C^{2}$. Let $\tilde{w}$ be a $W^{1}$ extension of $w$ to $\Omega_{1}$. We set $u=v-\bar{\partial} \tilde{w}$ in $\Omega_{1}$. Then $\mathrm{u}$ is in $L_{r, q-1}^{2}\left(\Omega_{1}\right)$ with $\bar{\partial} u=f$ in $\Omega_{1}$. But $u=0$ on $\Omega_{2}$. This implies that $u \in \operatorname{dom} \bar{\partial}_{\text {mix }}$ and $\bar{\partial}_{\text {mix }} u=f$.

Theorem 7.2. Let $\Omega=\Omega_{1} \backslash \bar{\Omega}_{2}$ between two bounded strictly pseudoconvex domains $\Omega_{1}$ and $\Omega_{2}$ in an $n$-dimensional Stein manifold $X$ such that $\Omega_{2} \Subset \Omega_{1}$. Then

$$
H_{\bar{\partial}_{\text {mix }}, L^{2}}^{r, 1}(\Omega) \neq 0 \text {. }
$$

Proof. The proof follows as in Lemma 2.3 in Li and Shaw [16]. 


\section{REFERENCES}

[1] O. Abdelkader and S. Saber, Solution to $\bar{\partial}$-equations with exact support on pseudoconvex manifolds, Int. J. Geom. Methods Mod. Phys. 4 (2007), 339-348.

[2] J. Cao, M. C.-Shaw and L Wang, Estimates for the $\bar{\partial}-$ Neumann problem and nonexistence of $C^{2}$ Levi-flat hypersurfaces in $\mathbb{P}^{n}$, Math. Z. 248 (2004), 183-221.

[3] D. Catlin, Sufficient conditions for the extension of CR structures, J. Geom. Anal. 4 (1994), $467-538$.

[4] D. W. Catlin and S. Cho, Extension of CR structures on three dimensional compact pseudoconvex CR manifolds, Math. Ann. 334(2) (2006), 253-280.

[5] S.-C. Chen and M.-C. Shaw, Partial Differential Equations in Several Complex Variables, AMS/IP Stud. Adv. Math. 19, Amer. Math. Soc., Providence, R.I., 2001.

[6] S. Cho, Extension of $C R$ structures on pseudoconvex CR manifolds with one degenerate eigenvalue, Tohoku Math. J. 55(3) (2003), 321-360.

[7] P. W. Darko, The $L^{2}-\bar{\partial}-$ problem on manifolds with piecewise strictly pseudoconvex boundaries, Math. Proc. Cambridge Philos. Soc. (1994), 116-147.

[8] G. M. Henkin and A. Iordan, Regularity of $\bar{\partial}$ on pseudococave compacts and applications, Asian J. Math. 4 (2000), 855-884.

[9] L. Hörmander, $L^{2}$-estimates and existence theorems for the $\bar{\partial}$-operator, Acta Math. 113 (1965), 89-152.

[10] L. Ho, $\bar{\partial}$-problem on weakly q-convex domains, Math. Ann. 290 (1991), 3-18.

[11] X. Huang and X. Li, $\bar{\partial}$-equation on a lunar domain with mixed boundary conditions, Trans. Amer. Math. Soc. 368(10) (2016), 6915-6937.

[12] J. J. Kohn, Harmonic integrals on strongly pseudoconvex manifolds, I, Ann. of Math. 78 (1963), 112-148.

[13] J. J. Kohn and H. Rossi, On the extension of holomorphic functions from the boundary of a complex manifold, Ann. of Math. 81 (1965), 451-472.

[14] J. J. Kohn, Global regularity for $\bar{\partial}$ on weakly pseudo-convex manifolds, Trans. Amer. Math. Soc. 181 (1973), 273-292.

[15] C. Laurent-Thiébaut and M.-C. Shaw, On the Hausdorff property of some Dolbeault cohomology groups, Math. Z. 274 (2013), 1165-1176.

[16] X. Li and M.-C. Shaw, The $\bar{\partial}$-equation on an annulus with mixed boundary conditions, Bull. Inst. Math. Acad. Sin. (N.S.) 8(3) (2013), 399-411.

[17] K. Matsumoto, Pseudoconvex domains of general order and q-convex domains in the complex projective space, Kyoto J. Math. 33 (1993), 685-695.

[18] S. Saber, Solution to $\bar{\partial}$ problem with exact support and regularity for the $\bar{\partial}-$ Neumann operator on weakly q-convex domains, Int. J. Geom. Methods Mod. Phys. 7(1) (2010), 135-142.

[19] S. Saber, The $\bar{\partial}$ problem on q-pseudoconvex domains with applications, Math. Slovaca 63(3) (2013), 521-530.

[20] S. Saber, The $\bar{\partial}$-problem with support conditions and pseudoconvexity of general order in Kähler manifolds, J. Korean Math. Soc. 53(6) (2016), 1211-1223.

[21] S. Saber, Compactness of the weighted $\bar{\partial}-$ Neumann operator and commutators of the Bergman projection with continuous functions, J. Geom. Phys. 138 (2019), 194-205.

[22] Y. T. Siu, Complex-analyticity of harmonic maps, vanishing and Lefschetz theorems, J. Differential Geom. 17 (1982), 55-138.

[23] H. H. Wu, The Bochner Technique in Differential Geometry, Harwood Academic, New York, 1988 . 
${ }^{1}$ Department of Mathematics,

FACUlty of Science And Arts in Baluurashi,

Albaha University,

Albaha, Saudi Arabia

${ }^{2}$ Department of Mathematics and Computer Science,

Faculty of Science, Beni-Suef University,

Beni Suef, EgYPT

Email address: sayedkay@yahoo.com 